\title{
Acciones educativas interculturales en educación superior
}

\author{
Araceli Martínez Arroyo \\ Dirección de Servicios para la Formación Integral \\ Universidad Iberoamericana Ciudad de México, MÉxico \\ araceli.martinez@ibero.mx
}

\section{2}

El mundo no es el mismo hoy que sale a la luz este número 76 de la revista $D I D A C$ del que fue hace unos meses cuando se abrió la convocatoria para la recepción de sus artículos. La contingencia ocasionada por el Covid-19 ha sido un evento global que ha trastocado certezas individuales y sociales y que, de manera urgente, nos convoca a mantener vivas y resignificar las teorías y las acciones que apuestan por la inclusión y la justicia social.

En este sentido, los once artículos de esta edición ilustran enfoques teóricos y prácticas que, desde el contexto universitario concretan procesos, acciones y relaciones interculturales de negociación, respeto y búsqueda de simetría en el diálogo. Su lectura en este momento, permite apreciar el camino andado y, al mismo tiempo, anima a no perderlo de vista y mantenerlo en el horizonte.

El primer artículo, "El acompañamiento tutorial en escritura académica universitaria como práctica intercultural”, ilustra la (re)construcción como sujetos científicos de 10 estudiantes indígenas que se percibían a sí mismos con un nivel académico deficiente y con habilidades de lectura y escritura por debajo del resto de sus compañeros. En esta experiencia, el aprendizaje del discurso académico se vincula con la experiencia personal identificando rasgos comunes entre las prácticas sociales universitarias y las prácticas culturales y lingüísticas de la comunidad de origen. De esta manera, ambos con- textos no se asumen como disociados entre sí, sino que se integran en la identidad del estudiante como un todo en el que los textos científicos cobran sentido desde la vivencia. Karla Susana Lombardi González y Juan Carlos Silas Casillas comprueban que este marco de interculturalidad de construcción de significados asumido en la tutoría para la promoción de la escritura académica se convierte en un acto dialógico y no meramente individual.

En "Formación de estudiantes indígenas universitarios en contextos citadinos mexicanos. Retos de la educación intercultural”, Elizabeth Martínez Buenabad y Ruth Belinda Bustos Córdova realizan una exploración de los procesos de ingreso, permanencia y continuidad de estudiantes indígenas en la Benemérita Universidad Autónoma de Puebla (BUAP) y en la Universidad Pedagógica Nacional (UPN) Unidad 171. Si bien alertan sobre los problemas que enfrentan aquellos alumnos que migran a la capital de su estado para realizar estudios universitarios, también señalan acciones realizadas en ambas instituciones para su solución: documentación de experiencias escolares, talleres de elección de carrera o grupos de investigación.

La investigación de Sergio Iván Navarro Martínez y Antonio Saldívar Moreno expone algunas contradicciones en los procesos de formación de una universidad intercultural. Si bien señala que en México estas instituciones han tenido un papel pro-

Acciones educativas interculturales en educación superior. $\bullet 3$ Araceli Martínez Arroyo. DIDAC 76 (2020): 3-6 
tagónico en la gestión de la diversidad, también muestra que en el decir de docentes y estudiantes de la institución analizada, existen prácticas que aún no conducen a una cabal comprensión de la perspectiva de las universidades interculturales como práctica y apuesta política. El artículo "El papel de la interculturalidad en la construcción de sujetos identitarios. La experiencia de la Universidad Intercultural de Chiapas en el municipio de Oxchuc (UNICH-Oxchuc)" cuestiona prácticas cotidianas de la universidad intercultural en las que prevalece una equivalencia de lo intercultural con el uso y aprendizaje de la lengua, el reforzamiento identitario o ciertos esencialismos sobre la cultura. Los autores cuestionan que la reivindicación de un sujeto identitario resta la posibilidad de construir un sujeto político que cuestiona las relaciones de poder entre las culturas y busca la transformación de las formas históricas de relación injusta entre la cultura nacional y la de los pueblos originarios.

El artículo de Erica González Apodaca, "Rutas y significados de la profesionalización indígena. Experiencias de profesionistas Ayuujk en la educación superior" expone el significado y la toma de decisiones de hombres y mujeres en el tránsito de su formación profesional. Los cuatro ejes analíticos que sustentan la investigación (esfuerzo personal, familia y capital social; contexto de estudio; acceso a políticas afirmativas, y; pertenencia comunitaria) dan cuenta de los imaginarios que las comunidades educativas y los sujetos construyen en la profesionalización y que, argumenta la autora, deben ser reconocidos en la definición de políticas educativas del Estado.

En "La licenciatura en Turismo Alternativo de la Universidad Intercultural Maya de Quintana Roo (UIMQROo), desde la mirada intercultural de los egresados", Cecilia del Socorro Medina Martín, Margarita de Abril Navarro Favela y Andrea Danilu Uc Angulo presentan los resultados de un estudio en 23 egresados de este programa académico. La finalidad de la indagación es evaluar el aprendizaje adquirido durante la formación universitaria en relación con su situación laboral vigente. Las autoras destacan que más de la mitad de estos egresados se desempeñan en labores docentes y que cerca de la tercera parte labora en el campo del turismo. En ambos casos, los egresados aprecian la formación teórica y práctica recibida en cuanto a vinculación comunitaria, investigación, elaboración de proyectos, búsqueda de información, así como control de grupos y conocimiento de aves y fauna. Los resultados de la indagación permiten observar que la formación profesional obtenida posibilitó que la mayoría de estos egresados pudieran incorporarse al campo laboral; no obstante, las autoras advierten el reto que tiene la profesión de turismo alternativo de garantizar no solamente el ingreso económico de sus egresados, sino de alcanzar un impacto en los ámbitos sociales, culturales, políticos y lingüísticos vinculados con las comunidades mayas.

En "Actitudes de negación, reconocimiento, compartencia y de aprendizaje interlingüístico e intercultural en la Escuela Normal Bilingüe e Intercultural de Oaxaca (enBio)", Hugo Pacheco Sánchez analiza 47 relatos autobiográficos de estudiantes indígenas para conocer sus opiniones respecto a sus lenguas y variantes frente a otras lenguas y hablantes diferentes a las de su origen, así como la influencia de dichas actitudes en el desarrollo de diversos procesos formativos. Los resultados demuestran un proceso gradual que comienza con la negación a hablar la propia lengua por haber sufrido discriminación al hacerlo y culmina en la compartencia y el aprendizaje lingüístico. La conciencia lingüística como un proceso de construcción de identidad personal y colectiva se hace evidente en los relatos ofrecidos.

En un abordaje a partir de las voces de los sujetos, especialmente el de las mujeres, el artículo "Incorporación de mujeres indígenas mexicanas en la academia universitaria" de Norma Molina Fuentes reporta el resultado de 14 entrevistas aplicadas a académicas que se autoadscriben como parte de un pueblo indígena maya. La indagación ofrece un panorama de saberes y prácticas recurrentes en el ejercicio de la profesión académica; profesión a la que accede un porcentaje mínimo de la población y cuyos mecanismos excluyentes se acentúan aún más 
en la población indígena. Las mujeres del estudio -doce con estudios de posgrado, nueve hablantes de su lengua, tres con hijos y, en su totalidad migrantes con el propósito de continuar sus estudios- realizan una crítica desde sus disciplinas por la forma en que las universidades han llevado a cabo la investigación sobre los pueblos indígenas y en las que parece haber un discurso de falsa interculturalidad, en el que los pueblos son los que tienen que ser interculturales, pero no el resto de la población. Las entrevistadas alertan sobre los mecanismos de evaluación de la producción científica que no considera el trabajo comunitario como un rubro visibilizado. Si bien el artículo da cuenta de la valoración positiva que este grupo de académicas encuentra en dicha profesión, resulta una evidencia de las resistencias epistemológicas de las IEs ante la interculturalidad y, sobre todo, de los retos que aún se plantean en la tríada universidad-academia-comunidad.

Daniel Mato en su artículo "Los desafíos del racismo a la gestión, docencia, investigación y vinculación social en la educación superior" enfatiza el compromiso que las IEs tienen en el avance de la erradicación del racismo. El autor expresa que, si bien los problemas de inequidad y exclusión tienen su origen en factores socialmente estructurales que están más allá del ámbito del sistema de educación superior (acceso a la educación, la salud y la justicia, por ejemplo) en lo que es relativamente poco lo que se puede hacer desde las universidades, es en un proyecto de largo aliento donde estas instituciones realizan su contribución. La formación de técnicos y profesionales que ocupen posiciones sociales, políticas y económicas de gran incidencia es el lugar de acción de las universidades. En este sentido, la docencia, la investigación y la vinculación social deben mantener esta mirada. La oferta académica, así como prácticas que permitan el acceso de docentes altamente calificados, aunque éstos carezcan de título universitario, el desarrollo de prácticas en espacios sociales extrauniversitarios y la colaboración con diversos tipos de comunidades son ejemplos de acciones que, sin duda, abren caminos de conocimiento, sensibilización y compromiso.
En "Breve panorama histórico de la educación intercultural en América Latina" Jocelin Venegas Martínez y Luz María Stella Moreno Medrano exponen diversas propuestas educativas que desde los primeros ańos del siglo xx hasta el momento actual, concretan proyectos interculturales. De las primeras experiencias de educación bilingüe promovidas en América Latina en el siglo XvI por los misioneros, la Casa del Estudiante Indígena —en México-, los proyectos educativos que surgen en la pedagogía liberadora de Paulo Freire, hasta las recientes prácticas educativas emancipadoras que se gestan con la llamada "pedagogía decolonial". El artículo expone los retos de la conceptualización de lo intercultural y su concreción en prácticas educativas. Las autoras plantean el gran reto de visibilizar y deconstruir los procesos de colonización, así como conocer los mecanismos que usa el sistema para seguir oprimiendo a los pueblos indígenas. En este sentido, los planteamientos de la interculturalidad crítica se consideran vitales como herramienta pedagógica que cuestione el sistema, las relaciones de poder y las formas en que nos vinculamos con los otros.

"El enfoque intercultural en la Universidad Iberoamericana Ciudad de México" es, como su título indica, un planteamiento elaborado desde esta institución. Casandra Guajardo Rodríguez, Eugenia Legorreta Maldonado, Luz María Stella Moreno Medrano y Stefano Claudio Sartorello describen una propuesta de transversalización de la interculturalidad en los planes de estudio de licenciatura de dicha universidad. Los autores toman como base un planteamiento de la interculturalidad desde una perspectiva crítica y decolonial que permita comprender la realidad desde diferentes racionalidades y lógicas de pensamiento. Son cuatro elementos los que, a su juicio, constituyen esta perspectiva: intercultural, transcultural, interepistémica y, finalmente, la incidencia intra e intercultural. La propuesta de transversalización descrita encuentra su concreción en los planes de estudio principalmente, en el perfil de egreso de los programas académicos, en el desarrollo de competencias, en contenidos de asignaturas, en actividades curriculares y no curricula- 
res, así como en prácticas docentes. La implementación de dicho enfoque se sustenta en la formación de una comunidad crítica de docentes y estudiantes capaces de autorreflexionar sus propias prácticas y de formular nuevas estrategias de relaciones horizontales.

Finalmente, el artículo "Educación y formación interculturales como herramientas para la incidencia" describe dos proyectos de educación y formación intercultural en los que participa el Programa de Interculturalidad y Asuntos Indígenas (PIAI) de la Universidad Iberoamericana Ciudad de México: por un lado, el Programa de Intercambio Ibero-Instituto Superior Intercultural Ayuuk y, por otro lado, el Seminario sobre Resistencias y Alternativas. El primero de estos proyectos, se lleva a cabo entre estas dos instituciones (Ibero Ciudad de México e ISIA, con sede en Oaxaca). Los estudiantes de los diversos programas realizan una estancia semestral con una lógica de solución de problemas prácticos en el diseño de proyectos profesionales. La experiencia reportada por Eugenia Legorreta Maldonado y Sebastián Gabriel Olvera Gutiérrez, da cuenta del tránsito de estudiantes indígenas del ISIA que en un contexto urbano se confrontan con dinámicas académicas distintas de las de la escuela de origen (evaluación con exámenes y no con proyectos de impacto comunitario, por ejemplo). El segundo proyecto descrito, el Seminario sobre Resistencias y Alternativas es un espacio de reflexión colectiva entre el PIAI, la organización Servicios Integrales Émuri (sINÉ), el Instituto Intercultural Ñöñho (IIÑ) y el Programa Indígena Intercultural del Instituto Tecnológico y de Estudios Superiores de Occidente (ITESO). Dicho espacio resulta un foro al que se suman estudiantes que fortalecen su formación con las discusiones políticas, legales, culturales o económicas. Estas iniciativas, son ejemplos concretos en los que el PIAI cumple con su misión de contribuir en la transformación de las relaciones de desigualdad en contextos indígenas.

Las experiencias e investigaciones presentadas dan cuenta de escenarios diversos y de las necesidades y retos compartidos entre las universidades generales e interculturales, así como en las escuelas normales bilingües. Los enfoques, actores y procesos indagados pretenden abonar a la discusión sobre este tema de relevancia para las instituciones de educación superior.

En la edición de este número 76 expresamos un agradecimiento especial a la doctora Luz María Stella Moreno Medrano quien lo coordinó y ofreció un enfoque crítico en la comprensión de la educación intercultural.

\section{SEMBLANZa CURRICULAR}

Doctora en Educación y licenciada en Pedagogía por la Universidad Complutense de Madrid. Maestra y licenciada en Pedagogía por la Universidad Nacional Autónoma de México. Especialista en entornos virtuales de aprendizaje por la Organización de Estados Iberoamericanos. Ha participado en actividades de edición de contenidos y recursos educativos, investigación pedagógica, formación docente y desarrollo curricular.

Actualmente es académica de tiempo completo adscrita a la Dirección de Servicios para la Formación Integral de la Universidad Iberoamericana. Es miembro del Comité de Planes de

Estudio de Licenciatura y de Posgrado de esta institución, responsable del proyecto de investigación "La dimensión social en el curriculum de la Universidad Iberoamericana" y editora de la revista $D I D A C$. 